University of Nebraska - Lincoln

DigitalCommons@University of Nebraska - Lincoln

$1-1-2006$

\title{
Electromechanical imaging of biomaterials by scanning probe microscopy
}

\author{
B. J. Rodriguez \\ Condensed Matter Sciences Division, Oak Ridge National Laboratory, Oak Ridge, TN, \\ brian.rodriguez@ucd.ie \\ Sergei V. Kalinin \\ Oak Ridge National Laboratory, sergei2@ornl.gov \\ J. Shin \\ Condensed Matter Sciences Division, Oak Ridge National Laboratory, Oak Ridge, TN
}

Stephen Jesse

Oak Ridge National Laboratory, sjesse@ornl.gov

V. Grichko

North Carolina State University, Raleigh

See next page for additional authors

Follow this and additional works at: https://digitalcommons.unl.edu/physicsgruverman

Part of the Physics Commons

Rodriguez, B. J.; Kalinin, Sergei V.; Shin, J.; Jesse, Stephen; Grichko, V.; Thundat, T.; Baddorf, Arthur P.; and Gruverman, Alexei, "Electromechanical imaging of biomaterials by scanning probe microscopy" (2006). Alexei Gruverman Publications. 34.

https://digitalcommons.unl.edu/physicsgruverman/34

This Article is brought to you for free and open access by the Research Papers in Physics and Astronomy at DigitalCommons@University of Nebraska - Lincoln. It has been accepted for inclusion in Alexei Gruverman Publications by an authorized administrator of DigitalCommons@University of Nebraska - Lincoln. 


\section{Authors}

B. J. Rodriguez, Sergei V. Kalinin, J. Shin, Stephen Jesse, V. Grichko, T. Thundat, Arthur P. Baddorf, and Alexei Gruverman

This article is available at DigitalCommons@University of Nebraska - Lincoln: https://digitalcommons.unl.edu/ physicsgruverman/34 


\title{
Electromechanical imaging of biomaterials by scanning probe microscopy
}

\author{
B.J. Rodriguez ${ }^{\text {a,b }}$, S.V. Kalinin ${ }^{\text {a,f,* }}$, J. Shin ${ }^{\text {a,c }}$, S. Jesse ${ }^{\text {a }}$, V. Grichko ${ }^{\text {, }}$, \\ T. Thundat ${ }^{\text {e }}$, A.P. Baddorf ${ }^{\text {a }}$, A. Gruverman ${ }^{\text {f,* }}$ \\ ${ }^{a}$ Condensed Matter Sciences Division, Oak Ridge National Laboratory, Oak Ridge, TN 37831, USA \\ ${ }^{\mathrm{b}}$ Department of Physics, North Carolina State University, Raleigh, NC 27695, USA \\ ${ }^{\mathrm{c}}$ Department of Physics and Astronomy, University of Tennessee, Knoxville, TN 37996, USA \\ ${ }^{\mathrm{d}}$ Department of Biochemistry, North Carolina State University, Raleigh, NC 27695, USA \\ ${ }^{\mathrm{e}}$ Life Sciences Division, Oak Ridge National Laboratory, Oak Ridge, TN 37831, USA \\ ${ }^{\mathrm{f}}$ Department of Materials Science and Engineering, North Carolina State University, Raleigh, NC 27695, USA
}

Received 16 July 2005; received in revised form 23 September 2005; accepted 4 October 2005

Available online 9 December 2005

\begin{abstract}
The majority of calcified and connective tissues possess complex hierarchical structure spanning the length scales from nanometers to millimeters. Understanding the biological functionality of these materials requires reliable methods for structural imaging on the nanoscale. Here, we demonstrate an approach for electromechanical imaging of the structure of biological samples on the length scales from tens of microns to nanometers using piezoresponse force microscopy (PFM), which utilizes the intrinsic piezoelectricity of biopolymers such as proteins and polysaccharides as the basis for high-resolution imaging. Nanostructural imaging of a variety of protein-based materials, including tooth, antler, and cartilage, is demonstrated. Visualization of protein fibrils with sub-10 nm spatial resolution in a human tooth is achieved. Given the near-ubiquitous presence of piezoelectricity in biological systems, PFM is suggested as a versatile tool for micro- and nanostructural imaging in both connective and calcified tissues.
\end{abstract}

(C) 2005 Elsevier Inc. All rights reserved.

Keywords: Scanning probe microscopy; Piezoresponse force microscopy; Nanoscale; Piezoelectricity; Calcified tissues; Connective tissues

Biological materials are composed of dissimilar structural elements arranged in a complex hierarchical structure, each level bringing new aspects to the overall properties of the material. For calcified tissues, hardness and fracture strength exhibited on a micrometer level is due to the staggered configuration of nanoscale platelets of hard hydroxyapatite (HAP) intertwined with fibrils of the soft collagen matrix. The relative arrangement of the fibrils controls the tissue development, as well as determines mechanical properties of bone, dentin, and cartilage (Claes et al., 1995; Martin and Boardman, 1993; Nalla et al., 2003; Wu and

\footnotetext{
* Corresponding authors.

E-mail addresses: rodriguezbj@ornl.gov (B.J. Rodriguez), sergei2@ ornl.gov (S.V. Kalinin), alexei_gruverman@ncsu.edu (A. Gruverman).
}

Herzog, 2002). Notably, these materials simultaneously achieve maximal theoretically possible values both for fracture strength and toughness, a combination that is unique to biological systems, and which has promoted the search for biomimetic materials as a way to improve the properties of artificial materials. On larger length scales, the mineralized fibrils are arranged in a complex hierarchical structure, giving rise to as many as seven levels of structural organization (Weiner and Wagner, 1998). This hierarchical organization is common to most hard tissues including wood, seashells, etc., as summarized by Ji and Gao (2004).

A number of techniques have been developed and employed to study the nanostructure of biosystems. Several approaches based on diffraction methods such as X-ray diffraction (Boote et al., 2004; Bigi et al., 2001) and small-angle 
X-ray scattering (Fratzl et al., 1996; Kinney et al., 2001) have been developed, which provide information on average structure within $\sim 10-100 \mu \mathrm{m}$ regions of the material. Information on local preferential molecular orientation can be obtained using polarized light optical microscopy (Bromage et al., 2003). More sophisticated approaches include microwave imaging (Osaki et al., 2002), infrared Fourier transform spectrometry (Camacho et al., 1999), and second harmonic generation optical microscopy (Yasui et al., 2004). In the last several years, confocal optical microscopy has also been used for 3D mapping of collagen microstructure (Wu et al., 2003). However, the spatial resolution of these techniques is always limited by the diffraction limit or probe size, and the structure and properties of complex structural elements, such as Haversian and Volkmann canals or cement lines in bones, cannot be determined. Alternative approaches for mapping the internal structure of calcified and connective tissues are based on electron microscopy techniques such as scanning electron microscopy and transmission electron microscopy (Landis, 1995; Rubin et al., 2003). However, while providing valuable information on the geometric structure, electron microscopy requires careful sample preparation (staining, fixation, etc.), is generally incompatible with in vitro imaging, and, finally, does not provide information on local mechanical or electromechanical properties.

In this paper, we demonstrate an approach for high-resolution imaging of the structure of calcified and connective tissues based on the detection of local piezoelectric behavior using scanning probe microscopy (SPM). Piezoresponse force microscopy (PFM) based on the detection of local electromechanical signal is used to image nanoscale structure in biological systems such as calcified and connective tissues, exemplified by tooth enamel and dentin, antler, and cartilage. PFM is compared to traditional SPM techniques, such as topographic imaging by atomic force microscopy $(\mathrm{AFM})$ and local elasticity imaging by atomic force acoustic microscopy (AFAM) and ultrasonic force microscopy (UFM), which theoretically can be expected to differentiate between collagen and HAP based on the local elastic properties. It is shown that unlike AFM and AFAM, where signals are controlled or strongly affected by surface topography, PFM provides reliable data on the nanostructure. Ultimately, imaging the internal structure of protein inclusions in the tooth enamel in the vicinity of the dentinenamel junction (DEJ) is demonstrated with sub- $10 \mathrm{~nm}$ resolution.

Applicability of PFM for biological imaging stems from the near-ubiquitous presence of piezoelectricity and more complex forms of electromechanical coupling. Performed more than 200 years ago, experiments on muscular contraction in a frog under an electric bias (Galvani, 1953) were the first observation of the electromechanical coupling effect. One of the most important manifestations of the electromechanical behavior is piezoelectricity, which stems from the non-centrosymmetric crystal structure of most biopolymers including cellulose, collagen, keratin, etc. Piezoelectric behavior has been observed in a variety of biological systems including bones (Anderson and Eriksson, 1970; Fukada and Yasuda, 1957; Lang, 1966; Yasuda, 1957), teeth (Marino and Gross, 1989), wood (Bazhenov, 1961; Fukada, 1955), and seashells (Fukada, 1995). It has been postulated that piezoelectric coupling, via mechanical stress that generates an electric potential, controls the mechanisms of local tissue development (Bassett, 1968; Marino and Becker, 1970). Understanding the relationship between physiologically generated electric fields and mechanical properties on the molecular, cellular, and tissue levels has become the main motivation of studying piezoelectricity in biological systems. However, strong orientation dependence of the piezoelectric signal, combined with the structural complexity of most biological systems, effectively precluded the quantitative nanoscale studies required to establish the biological role of piezoelectricity. Here, we demonstrate that probing local piezoelectricity allows the local structure of these materials to be probed on the nanoscale.

The local electromechanical properties are accessed by PFM (Gruverman, 2004; Kalinin et al., 2004). In this technique, the application of a periodic electrical bias, $V_{\mathrm{tip}}=V_{\mathrm{dc}}+V_{\mathrm{ac}} \cos \omega t$, between a conductive SPM tip and the backside of the sample, results in a periodic displacement of the surface, $d=d_{1 \omega} \cos (\omega t+\varphi)$, that can be measured with sub-Angstrom precision. The interaction volume beneath the tip (the volume that is piezoelectrically excited) depends on the contact radius, the applied bias, and local properties of the material, and is generally of the order of 5 $20 \mathrm{~nm}$, providing the measure of spatial resolution and field penetration in the material. The amplitude and phase of the cantilever oscillations reveal the information on the strength and sign of the local electromechanical response, respectively. Both vertical and lateral components of surface displacement are measured (Eng et al., 1999), providing information on normal and in-plane components of the electromechanical response vector. The image formation mechanism in PFM has been analyzed in detail (Kalinin et al., 2004) and it has been shown that in the absence of a dielectric gap between the tip and the surface, the PFM signal magnitude is independent of the contact area. The latter, however, determines the lateral spatial resolution. During the last decade, PFM has become the primary tool for the characterization of ferroelectric materials and selected piezoelectric materials, e.g., III-V nitrides (Rodriguez et al., 2002), on the nanoscale; however, until recently (Kalinin et al., 2005), information on local electromechanical properties in biological systems has been extremely limited (Halperin et al., 2004) and only the relatively macroscopic elements such as Haversian channels have been observed.

A complementary approach for structural imaging can be based on local mechanical scanning probe techniques such as AFAM (Rabe et al., 2002) and UFM (Yamanaka et al., 1994), which can be utilized to potentially distinguish dissimilar components of calcified tissues based on differences in mechanical properties. In AFAM, the sample is 
vibrated mechanically by a piezoelectric actuator, and acoustical waves transmitted to the tip are detected, providing a contrast between hard and soft regions of the sample. In UFM, a second voltage (at a different frequency) is applied to the actuator, allowing the detection signal and the driving signal to be separated. In both cases, in the small signal limit, the AFAM/UFM signal is related to the effective spring constant of the tip-surface junction, from which the elastic modulus of material can be determined. However, AFAM imaging of topographically inhomogeneous systems is limited by a significant topographic crosstalk due to the variations in local surface geometry (slope and local radius of curvature) that influences the contact mechanics of the tip-surface junction, thus precluding reliable elastic imaging on the nanoscale. Moreover, even for relatively flat surfaces, the AFAM signal is strongly related to the tip shape and radius of curvature, necessitating the use of calibration standards with known properties for quantitative measurements (Fig. 1). Conversely, the PFM signal is virtually insensitive to the tip geometry, provided that good contact between the tip and the surface is established, resulting in significantly less sensitivity of the technique to topographic cross-talk.

In general, successful PFM imaging requires a sharp conducting tip and a clean surface with minimal contamination. To establish the integrity of conductive coating, the quality of the tip is first verified with a known ferroelectric standard, such as lead zirconate-titanate (PZT) ceramics or periodically poled lithium niobate. In addition, to obtain quantitative information, the tip can be calibrated using an appropriate standard (Kalinin et al., accepted). Application of PFM to biological systems necessitated new routes for surface preparation to avoid a surface contamination layer. Here, we have found that dentin and enamel produce an uncontaminated surface by simple mechanical polishing. Other systems such as antler develop lipid layers that result in decay of signal with time, and require either rapid imaging after surface preparation, as done in this paper, or possibly solvent cleaning.
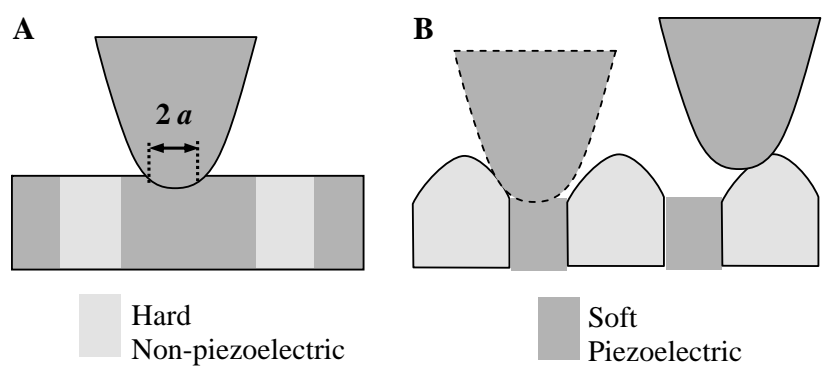

Fig. 1. The signal in AFAM and UFM is determined by the spring constant of the tip-surface junction, which is directly proportional to the contact radius. Thus, regions with different mechanical properties can be unambiguously distinguished only in the absence of topographic variations (A), while on a topographically inhomogeneous surface (B) changes in contact area due to local curvature will affect the AFAM signal. In comparison, the PFM signal is weakly dependent on the contact area and thus is insensitive to topographic cross-talk.
Here, we have employed an approach based on simultaneous acquisition of PFM and AFAM data that provides mechanical, electromechanical, and topographical images of biological systems with perfect spatial correlation (Shin et al., 2005). PFM and AFAM are implemented on a commercial SPM system (Veeco MultiMode NS-IIIA) equipped with additional function generators and lock-in amplifiers (DS 345 and SRS 830, Stanford Research Instruments, and Model 7280, Signal Recovery). A custom-built tip holder was used to allow direct tip biasing and to avoid capacitive cross-talk in the SPM electronics. Measurements were performed using $\mathrm{Pt}$ and $\mathrm{Au}$ coated tips (NSC-12 C, Micromasch, $l=130 \mu \mathrm{m}$, resonant frequency $\sim 150 \mathrm{kHz}$, spring constant $k \sim 4.5 \mathrm{~N} / \mathrm{m}$ ). Vertical PFM (VPFM) measurements were performed at frequencies $50-100 \mathrm{kHz}$, which minimizes the longitudinal contribution to the measured vertical signal. For lateral PFM (LPFM), the optimal conditions for contrast transfer are $\sim 10 \mathrm{kHz}$; for higher frequencies, the onset of sliding friction minimizes in-plane oscillation transfer between the tip and the surface (Yamanaka et al., 1994; A. Kholkin, unpublished data). For AFAM measurements, the samples were glued to a commercial PZT oscillator (Piezo Kinetics, Bellefonte, PA). To minimize cross-talk between PFM and AFAM signals, the top electrode of the oscillator was always grounded and the modulation bias was applied to the bottom electrode. Custom LabView software was developed for simultaneous acquisition of VPFM and LPFM phase and amplitude data and AFAM data, emulating additional SPM data acquisition channels. Note that while for well-known materials, acquisition of the PFM $x$-signal, $P R=d_{1 \omega} \cos \varphi$, will be sufficient, in this case, both amplitude and phase images were collected to establish the veracity of the data.

A PFM-AFAM approach has been used to perform mechanical and electromechanical imaging in a variety of biomaterials. The deciduous human tooth sample was cross-sectioned parallel to the growth direction and polished using diamond polishing pads down to $0.5 \mu \mathrm{m}$ grit size. The sample was subsequently mounted on the PZT oscillator using silver paint. A similar approach was used for antler sample preparation. Canine femoral cartilage was microtomed to a final thickness of $10 \mu \mathrm{m}$ and the resulting sliver was glued to the conductive sample holder. In all cases, PFM imaging of biomaterials was found to be extremely sensitive to surface preparation, with minute contaminations precluding successful imaging. However, simple approaches as described above often provide good results.

PFM and AFAM imaging of tooth structure are illustrated in Fig. 2. The outer layer of tooth, enamel, is comprised primarily by HAP crystals and small fraction $(\sim 3-5 \%)$ of organic proteins (including amelogenins and ameloblastins) concentrated in the vicinity of dentinenamel junction. The dentin layer below the enamel has a significantly higher fraction of organic material (predominantly collagen I), up to $30-40 \%$, and is formed by growth tubules that partially continue to the enamel layer. The 

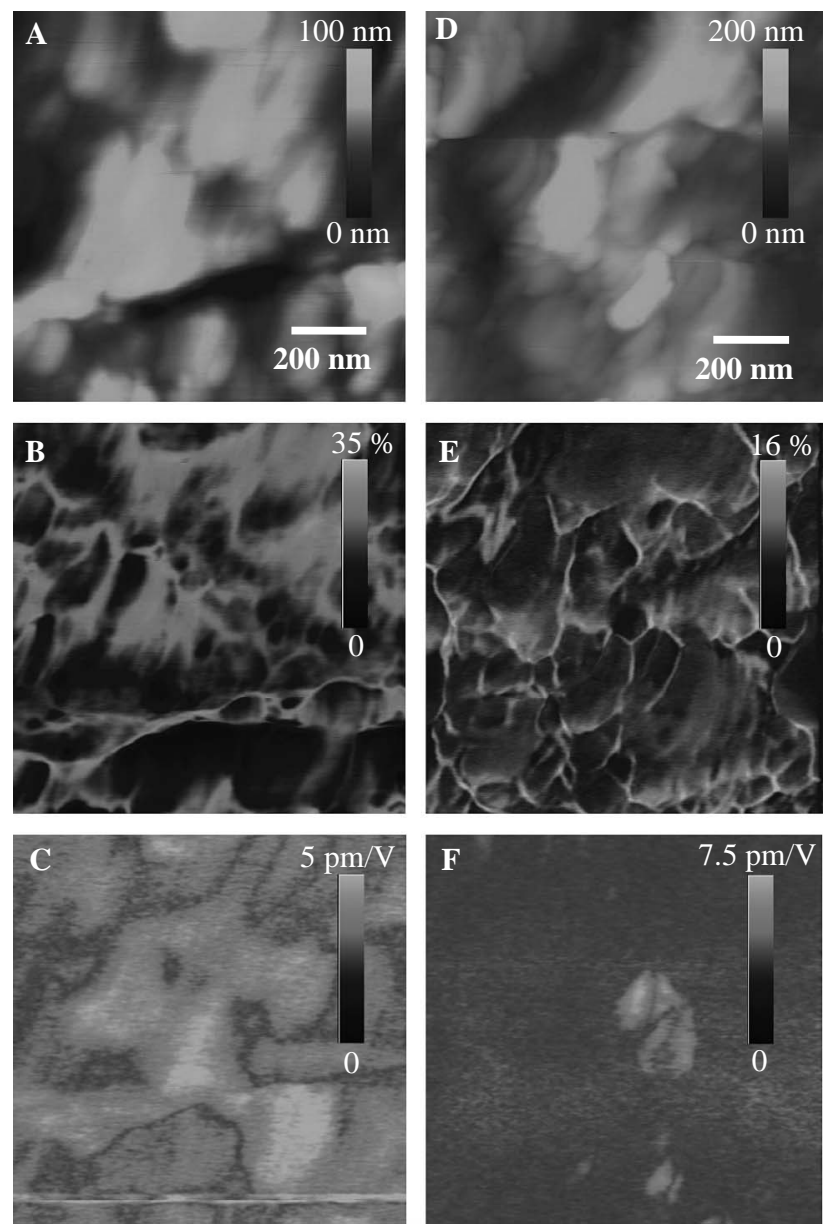

Fig. 2. Topographic (A and D), elastic (B and E), and piezoelectric (C and F) images of dentin $(A-C)$ and enamel (D-F) regions of dry tooth. Vertical scale for elastic images (B and E) is reported as a percentage of the average signal.

enamel and dentin regions can be readily identified using optical microscopy. Comparison of topographic images of the different dental tissues in Figs. 2A and D with corre- sponding elastic images (Figs. 2B and E), shows that elasticity measurements in SPM provide much more detailed information on the internal tooth structure and allow visualization of features invisible on the topographic image. However, while clear contrast between enamel and dentin was observed on a $10-\mu \mathrm{m}$ scale (not shown), on the nanoscale, the image is strongly affected by the topographic cross-talk when the grooves between the grains are associated with the bright features of elasticity images. Even though in some cases AFAM provides topography-independent contrast, in general, topographic cross-talk due to changes in local surface curvature precludes unambiguous differentiation of tissue components. In comparison, a marked difference between dissimilar dental tissues is observed in the VPFM images in Figs. 2C and F. A strong response signal of the dentin region is consistent with a high density of piezoelectrically active collagen (Marino and Gross, 1989). Interestingly, several isolated regions with a high-piezoresponse signal are observed in the enamel region, indicative of the presence of a low fraction of protein fibers. Although it is recognized that enamel of a fully developed tooth does not contain proteins, the early stages of tooth development require the presence of amelogenins to guide the HAP crystal growth (Habelitz et al., 2004; Ishiyama et al., 1999). No protein inclusions were observed in the outer layers of enamel.

In order to verify that the observed PFM contrast is indicative of the presence of protein, a sample of pure collagen I from a rat tail tendon has been imaged. The purified collagen was spin-cast onto a $\mathrm{Pt} / \mathrm{SiO}_{2} / \mathrm{Si}$ substrate. The topography, VPFM amplitude and phase are shown in Figs. 3A-C, respectively. This reconstituted collagen has not precipitated on the substrate in fibril form, however, as evidenced by the VPFM phase (Fig. 3C) there does appear to be some self-assembly, as the regions of uniform phase are larger than the topographic features. In Figs. 3D-F, topography, VPFM amplitude and phase are shown for a
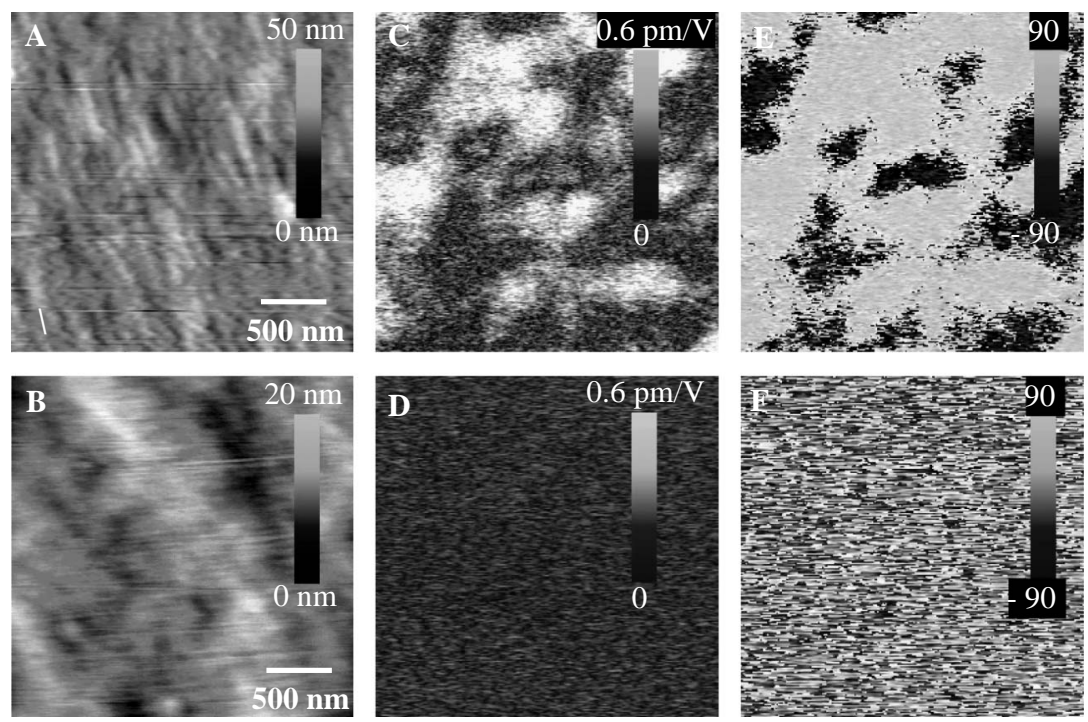

Fig. 3. Topographic (A and B), vertical PFM amplitude (C and D) and phase (E and F) images of type I collagen and $\mathrm{SiO}_{2}$, respectively. 

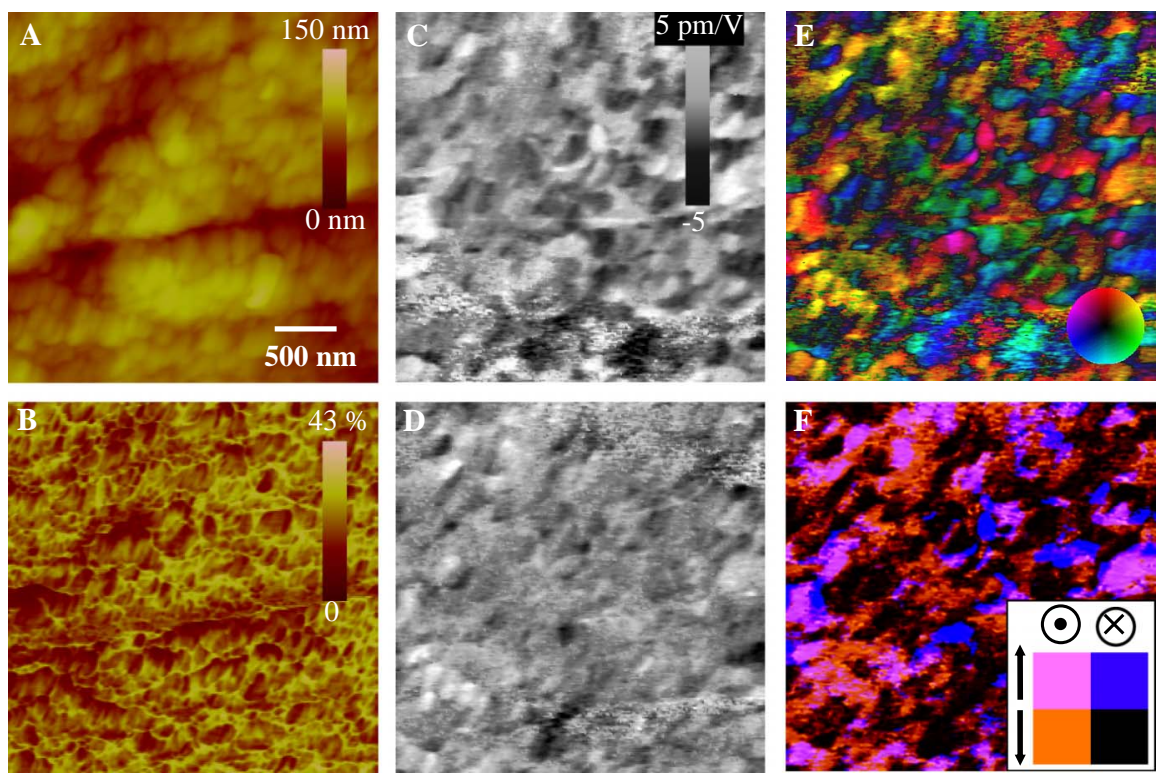

Fig. 4. (A) Topographic, (B) AFAM, (C) vertical PFM, and (D) lateral PFM images of dentin. (E) 2D (vpr,lpr) vector and (F) phase PFM maps (overlay of vertical and lateral PFM phase images). On the color wheel legend, color corresponds to the orientation, while color intensity corresponds to the magnitude (maximum is $5 \mathrm{pm} / \mathrm{V}$ ). The lateral PFM signal is not calibrated. (For interpretation of the references to color in this figure legend, the reader is referred to the web version of this paper.)

$\mathrm{SiO}_{2} / \mathrm{Si}$ substrate. The $\mathrm{SiO}_{2} / \mathrm{Si}$ sample shows no piezoelectric activity. HAP is also centrosymmetric and hence, nonpiezoelectric. Furthermore, in adult teeth cross-sections, no piezoresponse is observed from the enamel region (where nearly all protein has been processed). Thus, we conclude that the piezoresponse is due to the protein and not due to the HAP, in which crystal structure prohibits piezoelectricity.

As a further extension of this approach, PFM allows the determination of two independent components of the electromechanical response vector, collected as vertical and lateral PFM signals. Shown in Figs. 4A and B are topographic and AFAM images of the dentin region. In comparison, Figs. 4C and D, illustrate vertical and lateral PFM images, providing information on out-of-plane and in-plane electromechanical response. Note that unlike AFAM, there is no correlation between vertical PFM, lateral PFM data, and topography. To obtain further insight into the nanostructure of dentin, we employ a vector presentation for PFM. The VPFM and LPFM images are normalized with respect to the maximum and minimum values of the signal amplitude so that the intensity changes between -1 and 1 , i.e., $v p r, l p r \in(-1,1)$. Using commercial software (Mathematica, 5.0), this $2 \mathrm{D}$ vector data $(v p r, l p r)$ is converted to the amplitude/angle pair, $A_{2 \mathrm{D}}=\operatorname{Abs}(v p r+I l p r), \theta_{2 \mathrm{D}}=\operatorname{Arg}(v p r$ + I lpr). These data are plotted so that the color corresponds to the orientation, while color intensity corresponds to the magnitude, as shown in color wheel diagram. The approach for calibration is described elsewhere (Kalinin et al., accepted). Note that unlike conventional SPM when pseudocolor images are used to better represent scalar (height, current, etc.) images, here the color is used to represent the local vector field orientation. The color-encoded vector response map (vector PFM) is shown in Fig. 4E. Thus obtained image contains information on both the orientation (color) and the magnitude (brightness) of electromechanical response vector. Shown in comparison in Fig. $4 \mathrm{~F}$ is the phase overlay obtained from the superposition of vertical and lateral PFM phase images using Adobe Photoshop layer blending. Here, blue corresponds to a domain that is oriented toward the top of the figure and into the plane, orange corresponds to a domain oriented toward the bottom of the figure and out of the plane, black corresponds to into the plane and toward the bottom of the figure, and violet corresponds to out of the plane and towards the top of the figure. This phase image allows the correspondence between regions with different orientation to be established.

As a second example of elastic and electromechanical imaging, these techniques were used to study the structure of a deer antler. Shown in Fig. 5 are images of surface topography and UFM of the perpendicular antler crosssection. The topography exhibits a number of features presumably related to the surface preparation. The comple-
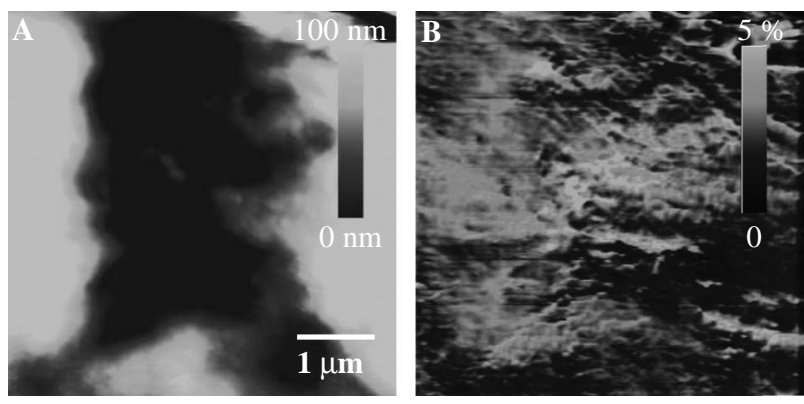

Fig. 5. Topographic (A) and ultrasonic (B) images of a deer antler. 
mentary UFM image illustrates local contrast variations across the image related to either topographic cross-talk or intrinsic property variation in the material. Note that the effective resolution in the UFM image is significantly higher than in topographic data, and in an independent experiment was shown to be $\sim 5 \mathrm{~nm}$, comparable with the tip-surface contact area estimated for the imaging conditions.

Fig. 6 illustrates surface topography (A) and piezoresponse (B and C) images of a longitudinal cross-section of the antler. Unlike teeth, this material contains lipids which can diffuse to the surface resulting in the formation of nonconductive layer which significantly complicates PFM imaging and necessitates the use of cantilevers with relatively high spring constants $(>5 \mathrm{~N} / \mathrm{m})$ to penetrate the contamination layer. To visualize electromechanical response data, we employ a vector representation for PFM as illustrated in Figs. 6B and C. Note that vector PFM allows clear visualization (arrow in Fig. 6B) of previously invisible microstructural elements, presumably a region with different keratin orientation. Vector PFM (Fig. 6C) of a smaller area clearly shows elongated keratin fibrils 2$3 \mu \mathrm{m}$ long and $200-300 \mathrm{~nm}$ wide (marked by white ellipses), oriented along the antler axis.

Finally, illustrated in Fig. 7 is a series of topographic and PFM images for canine cartilage. The surface is formed by multiple mounds with characteristic size of $100-200 \mathrm{~nm}$, formed presumably during drying and shrinking of the cartilage surface. No significant variations of AFAM signal (not shown) other than at the grooves were observed, sug- gesting that the surface is elastically homogeneous. At the same time, vertical PFM phase and amplitude images shown in Figs. 7B and C clearly illustrate that the surface is piezoelectric, with the piezoelectric domains associated with the mounds. Note that the use of relatively stiff cantilevers required to obtain reliable PFM signal on a relatively soft surface results both in low topographic resolution and presence of imaging artifacts (spurious lines in PFM images).

Data in Figs. 2, 4, 6, and 7 illustrate the applicability of PFM for structural characterization on micron and larger length scales in a broad range of materials system. To establish the resolution limit of this technique, the imaging was performed on an individual protein inclusion in tooth enamel. Shown in Fig. 8A is a topographic image of enamel in the vicinity of DEJ. Corresponding vertical and lateral PFM images in Figs. 8B and C show a strong electromechanical response that we attribute to a protein inclusion embedded within a non-piezoelectric matrix. The spatial resolution of PFM, determined as a half-width of the boundary between different piezoelectric regions, is about $5 \mathrm{~nm}$ (Kalinin et al., 2005). Note that the resolution achieved is of the order of magnitude better than 50 $100 \mathrm{~nm}$ typical for single crystals (Rodriguez et al., 2005) and is comparable to the best results achieved to date for thin films of ferroelectric perovskites.

Comparison of the VPFM and the LPFM images shows a different pattern of piezoelectric domains, suggesting a complicated structure of the protein inclusion, consisting of
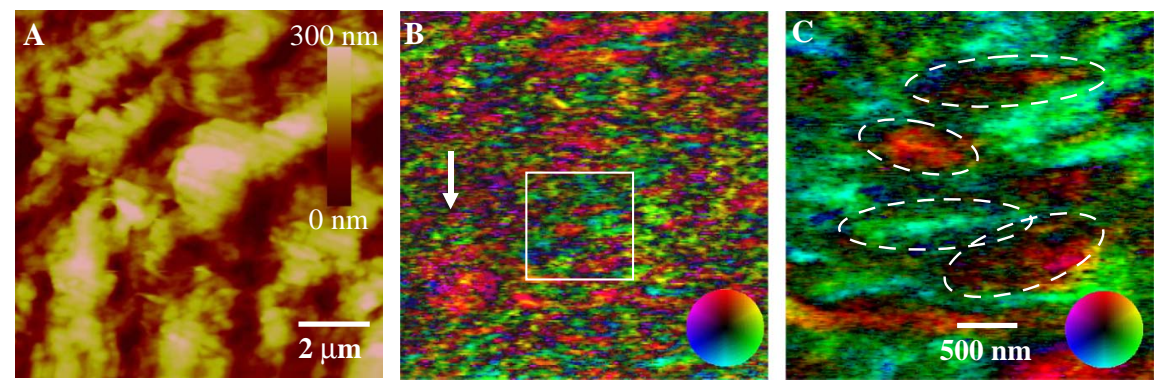

Fig. 6. Surface topography (A) and vector PFM images (B and C) of deer antler. On the color wheel legend, the color corresponds to the orientation of keratin fibers, while the color intensity corresponds to the magnitude (maximum is $4 \mathrm{pm} / \mathrm{V}$ ). Vector PFM illustrates finer details of internal antler structure, including the presence of region with different keratin orientation. The characteristic keratin fiber width in the PFM image is $\sim 200 \mathrm{~nm}$. Note that there is no correlation between PFM and topographic images, suggesting absence of cross-talk. (For interpretation of the references to color in this figure legend, the reader is referred to the web version of this paper.)
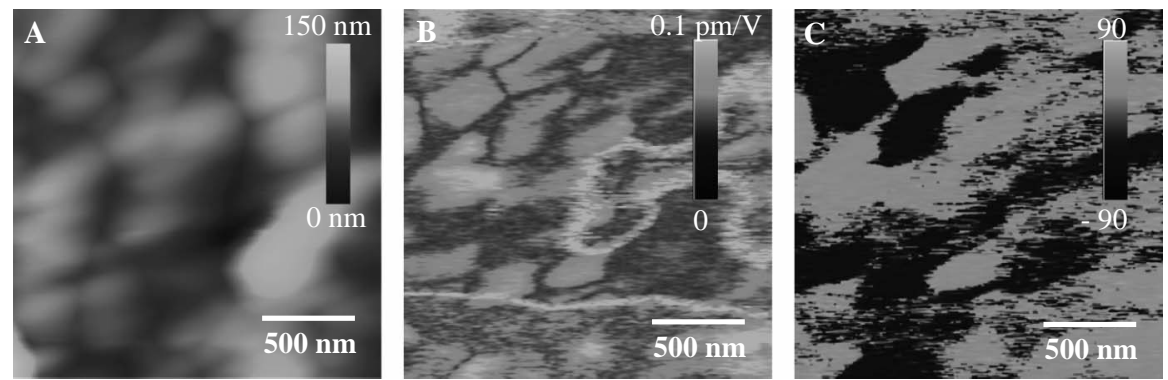

Fig. 7. (A) Surface topography and vertical PFM (B) amplitude and (C) phase images of the microtomed canine femoral cartilage. 


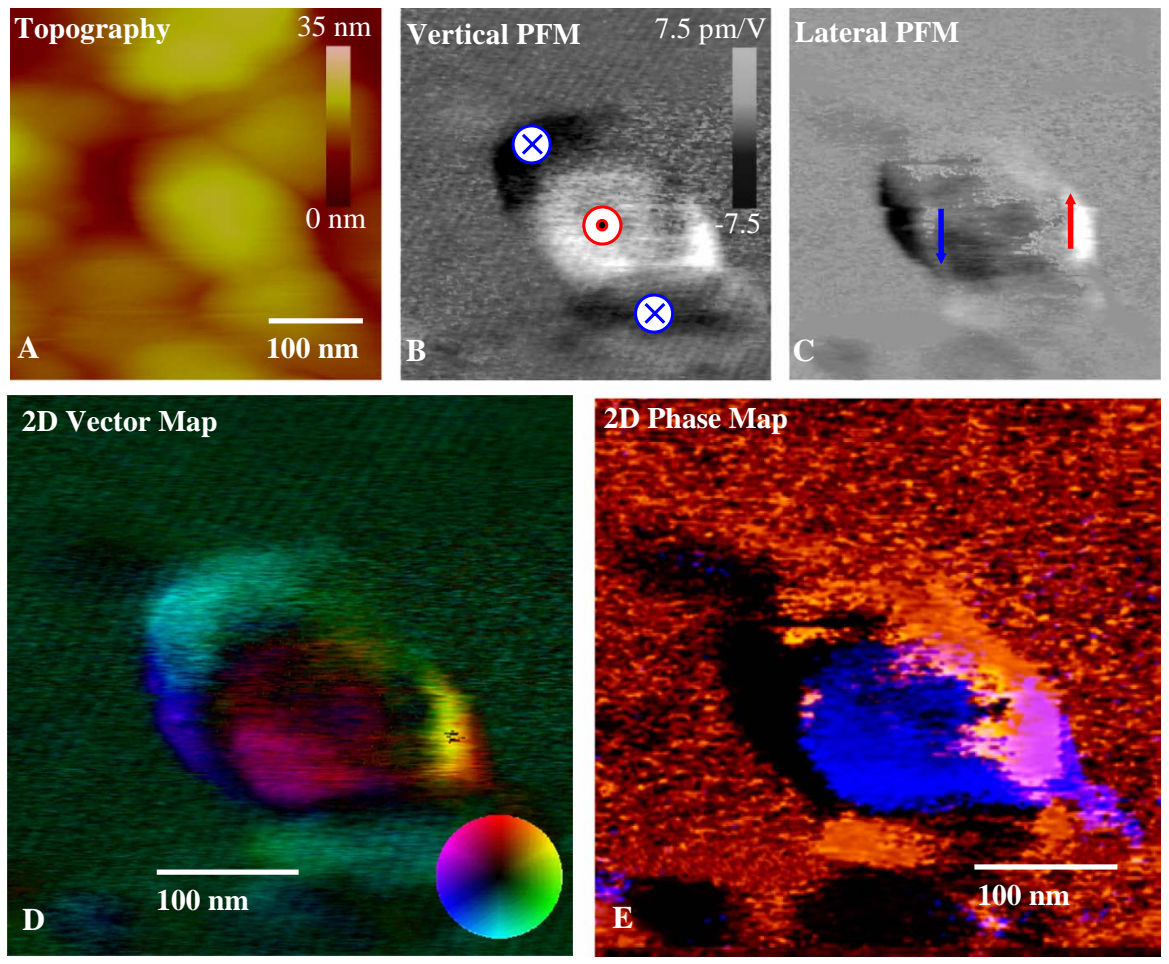

Fig. 8. (A) Surface topography (vertical scale $20 \mathrm{~nm}$ ), (B) vertical PFM and (C) lateral PFM images of protein inclusion in tooth enamel. (D) Vector PFM and (E) phase PFM maps of local electromechanical response. Color wheel (in (D)) indicates the orientation of the electromechanical response vector, while the intensity provides the magnitude (maximum is $7.5 \mathrm{pm} / \mathrm{V}$ ). (For interpretation of the references to color in this figure legend, the reader is referred to the web version of this paper.)

several microfibrils. The color-encoded vector response map (vector PFM), shown in Fig. 8D, clearly delineates the complex structure, visualizing the electromechanically active protein fibril conformation in real space (Smith, 1968). Corresponding phase images are illustrated in Fig. 8E, illustrating preferential piezoelectric orientations in the material.

To complement PFM imaging, Fig. 9 shows a local electromechanical response within a single 150 -nm protein fiber measured as a function of modulation bias. The tip oscillation amplitude is a nearly linear function of modulation bias, as expected for a piezoelectric material. For higher biases, the effective piezoresponse decreases, presumably due to the nonlinear signal transfer in the tip-surface junction. Alternative explanations such as onset of ionic conductivity or piezoelectric non-linearity of the biopolymer are inconsistent with the

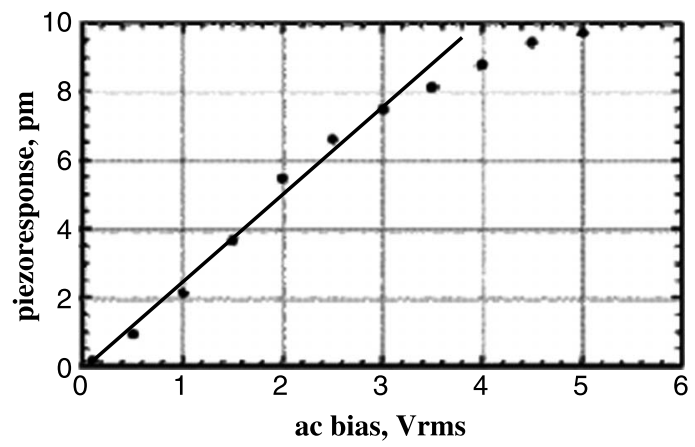

Fig. 9. Modulation bias dependence of the PFM signal within single $\sim 150 \mathrm{~nm}$ protein fibril yields an effective response of $\sim 2.5 \mathrm{pm} / \mathrm{V}$. results of local piezoelectric spectroscopy measurements. The effective piezoelectric coefficient is $d_{\text {local }}=1.5-2.5 \mathrm{pm} / \mathrm{V}$, significantly larger than $d=0.028 \mathrm{pm} / \mathrm{V}$ for a macroscopic dentin sample and comparable to $d=0.28 \mathrm{pm} / \mathrm{V}$ for dry bone (Marino and Gross, 1989). This illustrates that the partial disorder in calcified tissues such as bones can significantly reduce the macroscopic piezoelectric properties compared to the constituent fibrils. This observation also suggests that electromechanical coupling can be used as a measure of calcified tissue microstructure, an approach suggested for wood characterization in the 1960s (Bazhenov, 1961).

Finally, shown in Fig. 10 are phase and amplitude piezoelectric spectroscopy measurements obtained on a single protein inclusion and adjacent hydroxyapatite region. No inversion of the strain sign in protein (amplitude and phase are constant) upon application of the dc bias was observed, indicating that the material is piezoelectric, but not ferroelectric. In comparison, in the HAP region, the amplitude is virtually zero, and the phase changes sign at $\sim 2 \mathrm{~V}$, as expected for the case when PFM contrast is dominated by electrostatic forces.

To summarize, piezoelectric coupling in biomaterials can be used as a basis for high-resolution imaging on the length scales from tens of microns to $10 \mathrm{~nm}$, i.e., on the length scale of the individual building blocks of the structural properties. Unlike elasticity imaging by AFAM and UFM, the PFM signal is virtually independent on surface topography, allowing reliable imaging of material nanostructure with sub- $10 \mathrm{~nm}$ resolution. PFM has the potential 

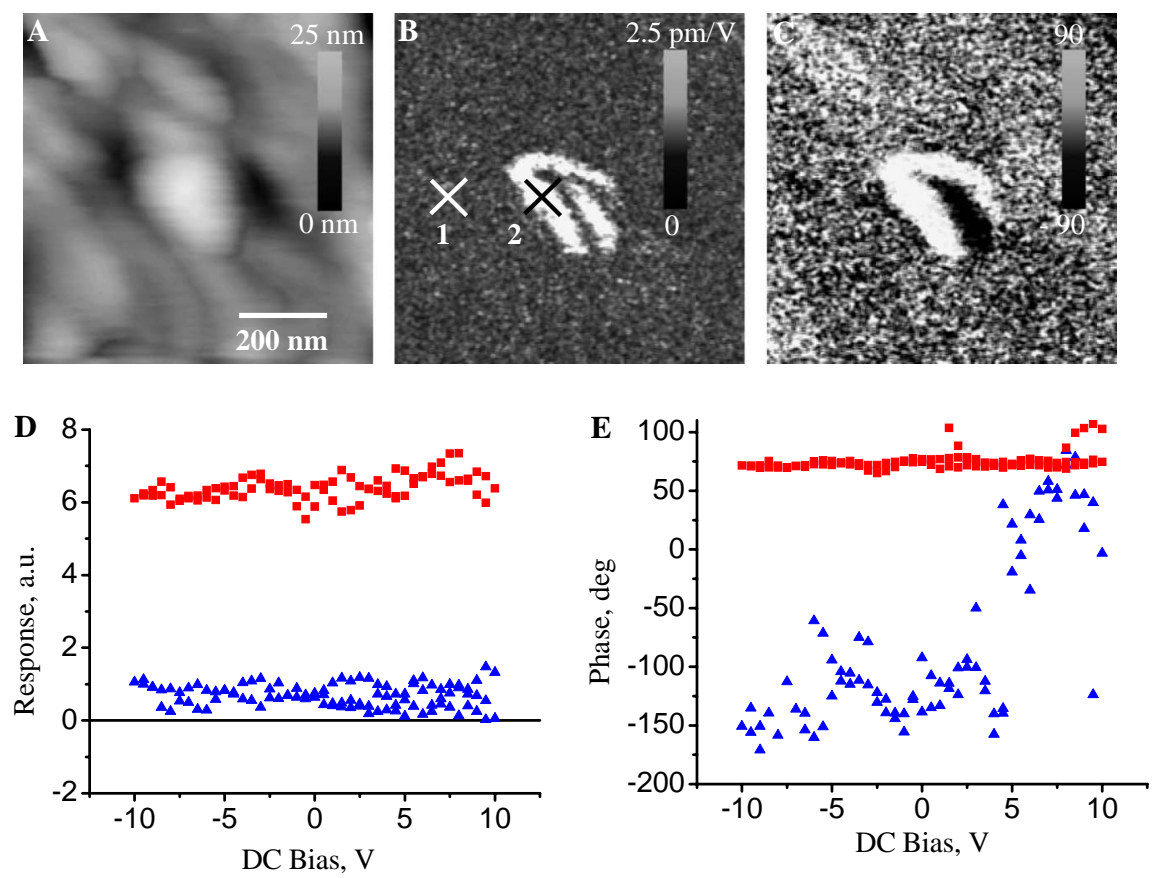

Fig. 10. (A) Surface topography and vertical PFM (B) amplitude and (C) phase of a single protein inclusion in the enamel. (D) Amplitude and (E) phase hysteresis loops of the protein $(\mathbf{\square})$ and adjacent hydroxyapatite surface $(\mathbf{A})$ acquired in locations 1 and 2. (For interpretation of the references to color in this figure legend, the reader is referred to the web version of this paper.)

to become a novel imaging tool for the characterization of calcified and connective tissues and possibly other biosystems on the nanometer level, thus improving the understanding of the structure-property-functionality relationship. PFM may also enable molecular orientation imaging as well as the development and testing of biologically inspired micromechanical devices.

\section{Acknowledgments}

Research performed in part as a Eugene P. Wigner Fellow and staff member at the Oak Ridge National Laboratory, managed by UT-Battelle, LLC, for the U.S. Department of Energy under Contract No. DE-AC0500OR22725 (S.V.K.). Support from ORNL SEED funding is acknowledged (S.V.K. and T.T.). A.G. acknowledges financial support of the National Science Foundation (Grant No. DMR02-35632). The authors acknowledge M. Yamauchi (UNC) and E. Loboa (NCSU) for collagen and cartilage samples, respectively.

\section{References}

Anderson, J.C., Eriksson, C., 1970. Nature 227, 491.

Bassett, C.A.L., 1968. Calcif. Tissue Res. 1, 252.

Bazhenov, V.A., 1961. Piezoelectric Properties of Wood. Consultants Bureau, New York.

Bigi, A., Burghammer, M., Falconi, R., Koch, M.H., Panzavolta, S., Riekel, C., 2001. J. Struct. Biol. 136, 137.

Boote, C., Dennis, S., Meek, K., 2004. J. Struct. Biol. 146, 359.

Bromage, T.G., Goldman, H.M., McFarlin, S.C., Warshaw, J., Boyde, A., Riggs, C.M., 2003. Anat. Rec. B New Anat. 274, 157.
Camacho, N.P., Rinnerthaler, S., Paschalis, E.P., Mendelsohn, R., Boskey, A.L., Fratzl, P., 1999. Bone 25, 287.

Claes, L.E., Wilke, H.J., Kiefer, H., 1995. J. Biomech. 28, 1377.

Eng, L.M., Guntherodt, H.-J., Schneider, G.A., Kopke, U., Saldana, J.M., 1999. Appl. Phys. Lett. 74, 233.

Fratzl, P., Schreiber, S., Klaushofer, K., 1996. Conn. Tissue Res. 35, 9

Fukada, E., 1955. J. Phys. Jpn. 10, 149.

Fukada, E., 1995. Biorheology 32 (6), 593.

Fukada, E., Yasuda, I., 1957. J. Phys. Soc. Jpn. 12, 1158.

Galvani, L., 1953. De Viribus Electricitatis in Motu Musculari Commentarius, in Antichi Commentari, Bologna (1791). Commentary on the effect of electricity on muscular motion. English translation by R.M. Green. Cambridge, MA.

Gruverman, A., 2004. In: Nalwa, H.S. (Ed.), Encyclopedia of Nanoscience and Nanotechnology, vol. 3. American Scientific Publishers, Los Angeles, pp. 359-375.

Habelitz, S., Kullar, A., Marshall, S.J., DenBesten, P.K., Balooch, M., Marshall, G.W., Li, W., 2004. J. Dent. Res. 83, 698.

Halperin, C., Mutchnik, S., Agronin, A., Molotskii, M., Urenski, P., Salai, M., Rosenman, G., 2004. NanoLetters 4, 1253.

Ishiyama, M., Inage, T., Shimokawa, H., 1999. Arch. Hist. Cyt. 62, 191.

Ji, B., Gao, H., 2004. J. Mech. Phys. Solids 52, 1963.

Kalinin, S.V., Karapetian, E., Kachanov, M., 2004. Phys. Rev. B 70, 184101.

Kalinin, S.V., Rodriguez, B.J., Jess, S., Thundat, T., Gruverman, A., 2005. Appl. Phys. Lett. 87, 053901.

Kalinin, S.V., Rodriguez, B.J., Jesse, S., Shin, J., Baddorf, A.P., Gupta, P., Jain, H., Williams, D.B., Gruverman, A., 2005. Microscopy and Microanalysis, accepted for publication.

Kinney, J.H., Pople, J.A., Marshall, G.W., Marshall, S.J., 2001. Calcif. Tissue Int. $69,31$.

Landis, W.J., 1995. Bone 16, 533.

Lang, S.B., 1966. Nature 5063, 704-705.

Marino, A.A., Becker, R.O., 1970. Nature 228, 473.

Marino, A.A., Gross, B.D., 1989. Arch. Oral Biol. 34 (7), 507.

Martin, R.B., Boardman, D.L., 1993. J. Biomech. 26, 1047.

Mathematica 5.0, Wolfram Research.

Nalla, R.K., Kinney, J.H., Ritchie, R.O., 2003. Biomaterials 24, 3955. 
Osaki, S., Tohno, S., Tohno, Y., Ohuchi, K., Takakura, Y., 2002. Anat. Rec. 266, 103.

Rabe, U., Kopycinska, M., Hiserkorn, S., Munoz-Saldana, J., Schneider, G.A., Arnold, W., 2002. J. Phys. D 35, 2621.

Rodriguez, B.J., Gruverman, A., Kingon, A.I., Nemanich, R.J., Ambacher, O., 2002. Appl. Phys. Lett. 80, 4166.

Rodriguez, B.J., Nemanich, R.J., Kingon, A., Gruverman, A., Kalinin, S.V., Terabe, K., Liu, X.Y., Kitamura, K., 2005. Appl. Phys. Lett. 86, 012906.

Rubin, M.A., Jasiuk, I., Taylor, J., Rubin, J., Ganey, T., Apkarian, R.P., 2003. Bone 33, 270.

SD 0.394-0.000-0.040-502, Piezo Kinetics, Bellefonte, PA 16823. www.piezo-kinetics.com.
Shin, J., Rodriguez, B.J., Baddorf, A.P., Thundat, T., Karapetian, E., Kachanov, M., Gruverman, A., Kalinin, S.V., 2005. J. Vac. Sci. Technol. B 23, 2102.

Smith, J.W., 1968. Nature 219, 157.

Weiner, S., Wagner, H.D., 1998. Annu. Rev. Mater. Res. 28, 271.

Wu, J.Z., Herzog, W., 2002. J. Biomech. 35, 931.

Wu, J., Rajwa, B., Filmer, D.L., Hoffmann, C.M., Yuan, B., Chiang, C., Sturgis, J., Robinson, J.P., 2003. J. Microsc. 210 (Pt. 2), 158.

Yamanaka, K., Ogiso, H., Kolosov, O., 1994. Appl. Phys. Lett. 64, 178.

Yasuda, L., 1957. J. Jpn. Orthop. Surg. Soc. 28, 267.

Yasui, T., Tohno, Y., Araki, T., 2004. Appl. Opt. 43, 2861. 